José Precioso'

Maria José Lopez"

José M Calheiros"'I

Manuel Macedo'

Carles Ariza"

Francesca Sanchez"

Anna Schiaffino ${ }^{v}$

Esteve Fernández ${ }^{v}$

Manel Nebot"

Instituto de Educação e Psicologia.

Universidade do Minho. Braga, Portugal

" Faculdade de Ciências da Saúde. Universidade da Beira Interior. Covilhã, Portugal

III Hospital de São Marcos. Braga, Portugal

Agência de Saúde Pública de Barcelona. Barcelona, Espanha

Instituto Catalão de Oncologia de Barcelona. Barcelona, Espanha

Correspondência | Correspondence: José Precioso

Instituto de Educação e Psicologia.

Universidade do Minho

Campus de Gualtar

4710-057 Braga, Portugal

E-mail: precioso@iep.uminho.pt

\section{Poluição do ar interior provocada pelo fumo do cigarro em locais públicos de Portugal*}

\author{
Indoor air pollution caused by \\ cigarette smoke in public places in \\ Portugal
}

\section{RESUMO}

OBJETIVO: Poucos têm sido os estudos para conhecer o grau de poluição pelo fumo do tabaco a que estão sujeitas as pessoas em vários lugares públicos e privados. O objectivo do estudo foi quantificar o nível de poluição do ar provocada pelo fumo do cigarro em locais de trabalho e de lazer.

MÉTODOS: O estudo foi realizado no concelho de Braga, Portugal, em 2005. A medição dos teores de nicotina no ar interior foi realizada com monitores passivos contendo um filtro de $37 \mathrm{~mm}$ de diâmetro tratado com bissulfato sódico no seu interior. Os monitores foram colocados em lugares públicos, de trabalho e de lazer, pré-definidos. Para cada um dos locais, calculou-se a mediana da nicotina.

RESULTADOS: A presença de nicotina foi detectada em $85 \%$ das amostras. Foram encontrados valores elevados de contaminação do ar nas discotecas, com mediana de $82,26 \mu \mathrm{g} / \mathrm{m}^{3}$, variando entre os 5,79 e os $106,31 \mu \mathrm{g} / \mathrm{m}^{3}$. Os locais de trabalho da administração pública e da universidade apresentaram os valores mais baixos de nicotina.

CONCLUSÕES: Os dados confirmam a necessidade de reforçar a implementação e sobretudo, o cumprimento de políticas sem fumo nos locais de trabalho e de lazer, em benefício da saúde dos trabalhadores e como medida reforçadora de um ambiente que facilite aos fumadores o abandono do fumo do tabaco.

DESCRITORES: Poluição por fumaça de tabaco, análise. Poluição do ar em ambientes fechados. Amostras de ar. Monitoramento do ar. Tabagismo, prevenção e controle. Portugal. 


\begin{abstract}
OBJECTIVE: There have been few studies investigating the level of cigarette smoke pollution to which people in several public and private places are exposed. The purpose of this study was to quantify the level of air pollution produced by cigarette smoking in workplaces and leisure settings.
\end{abstract}

METHODS: The study was carried out in Braga, Portugal, in 2005. Nicotine content in indoor air was measured using passive monitors containing a 37 $\mathrm{mm}$ diameter filter inside treated with sodium bisulphate. The monitors were installed in predefined public workplaces and leisure settings. Median nicotine content was estimated for each place studied.

RESULTS: Nicotine was detected in $85 \%$ of the samples. Extremely high air contamination levels were found in discos with a median of $82.26 \mu \mathrm{g} / \mathrm{m}^{3}$, ranging between 5.79 and $106.31 \mu \mathrm{g} / \mathrm{m}^{3}$. Workplaces of public administration and university buildings showed the lowest nicotine content.

CONCLUSIONS: The study findings confirm the need to promote the implementation of smoke-free policies in workplaces and leisure settings to protect workers' health and as a reinforcing measure of an environment which facilitates smokers to quit smoking.

KEY WORDS: Tobacco smoke pollution, analysis. Air pollution, indoor. Air samples. Air monitoring. Smoking, prevention \& control. Portugal.

\section{INTRODUÇÃO}

O "fumo ambiental do tabaco" (FAT), freqüentemente designado também por "tabagismo passivo" e "environmental tobacco smoke (ETS)" ou "secondhand smoke" para o idioma inglês, é hoje o principal e mais disseminado poluente presente no meio interior. Calcula-se que seja a terceira causa evitável de morte em alguns países desenvolvidos, depois do tabagismo activo e do alcoolismo. ${ }^{1}$

São inúmeros os estudos que têm mostrado a diversidade de efeitos adversos sobre a saúde dos não fumadores expostos ao FAT. Os primeiros estudos centraram-se na identificação dos riscos da exposição crónica das esposas dos fumadores. $\mathrm{O}$ estudo de Hirayama, baseado no seguimento de uma coorte de mulheres japonesas, concluiu a relação dose-resposta entre os hábitos tabágicos do marido e o risco de cancro pulmão. ${ }^{3}$

Segundo o International consultation on environmental tobacco smoke and child health, a OMS estima que cerca de 700 milhões, ou seja, quase metade das crianças de todo o mundo respiram ar poluído pelo fumo do tabaco, e que tal ocorre predominantemente em casa. $\mathrm{O}$ vasto número de crianças expostas, aliado à evidência que o FAT lhes provoca doença, constitui, assim, uma significativa ameaça à saúde pública. $^{8}$
Os níveis de exposição para os quais têm sido identificados efeitos carcinogénicos situam-se entre 1 a $10 \mu \mathrm{g} / \mathrm{m}^{3}$ de nicotina, ${ }^{9}$ produzindo morbilidade e mortalidade significativas. No que respeita às crianças estão documentados efeitos respiratórios agudos e crónicos em lares com fumadores (nicotina de 1 a $10 \mu \mathrm{g} / \mathrm{m}^{3}$ ) ou mesmo em lares com fumadores ocasionais (nicotina de 0,1 a $1,0 \mu \mathrm{g} / \mathrm{m}^{3}$ ). Ainda não seja possível estabelecer um limiar de exposição seguro e que o risco de uma pessoa que vive com um fumador desenvolver o cancro é de aproximadamente 1 em $1.000 .^{9}$

Na última década, em países como os Estados Unidos e Canadá proliferaram os esforços para restringir a prática de fumar em locais públicos. Tais medidas, embora com estratégias e resultados distintos, foram recentemente adoptadas por alguns países europeus.

Apesar da gravidade da exposição ao fumo ambiental do tabaco, sobretudo em crianças e adolescentes, são praticamente inexistentes os estudos realizados em Portugal sobre o tema.

O presente artigo é o primeiro estudo realizado em Portugal cujo objectivo foi quantificar os níveis de FAT em vários locais de trabalho públicos, empresas privadas e do sector da hotelaria. 


\section{MÉTODOS}

O estudo foi efectuado no âmbito do projecto "Descrição da situação actual, prévia à entrada em vigor, em Espanha, da nova Lei reguladora da venda, fornecimento, consumo e publicidade aos produtos tabágicos, para protecção da saúde da população e avaliação do impacto da nova Lei Reguladora da venda, da distribuição, do consumo e da publicidade dos produtos do tabaco para protecção da saúde da população". Esse projeto foi promovido pela Agência de Saúde Pública de Barcelona, tendo como participantes os departamentos de saúde de oito comunidades autónomas de Espanha (Catalunha, Comunidade Valenciana, Madrid, Galiza, La Rioja, Cantábria, Baleares e Estremadura) e a Universidade do Minho em Portugal.

A concentração de nicotina existente no ar de um ambiente interior é uma medida objectiva do nível de fumo de tabaco que seria inalado por uma pessoa no ambiente.

A nicotina no ar foi medida com o auxílio de monitores passivos com filtro de $37 \mathrm{~mm}$ de diâmetro tratado com bissulfato sódico, procedimento adoptado em estudos efectuados por López et $\mathrm{al}^{4} \mathrm{e}$ por outros investigadores. ${ }^{2,5}$ Os monitores foram colocados em lugares pré- definidos no protocolo do estudo e apresentados na Tabela. Foram recolhidas 50 amostras entre outubro e dezembro de 2005.

A amostra foi de conveniência, segundo critério de oportunidade e acessibilidade dos investigadores, e a disponibilidade e consentimento dos responsáveis por cada local.

Nas discotecas, os monitores foram suspensos à roupa de um colaborador, (na parte superior do tronco), com o filtro voltado para frente. Nos demais locais, os monitores foram suspensos no tecto, de forma que $o$ ar passasse através do filtro, tendo sido colocados em zonas centrais longe de fontes directas de fumo, como cinzeiros. Nas discotecas o período de exposição foi no mínimo de três horas; nos demais locais, durante sete dias.

Durante o tempo de exposição, o filtro não necessitou de nenhum tipo de manipulação. Finalizado o período de exposição, o filtro foi retirado e analisado no Laboratório da Agência de Saúde Pública de Barcelona, mediante cromatografia de gases com limite de deteç̧ão de $0,01 \mu \mathrm{g}$.

Informações com o código da amostra, local, datas de colocação e retirada do monitor, área e volume do local

Tabela. Locais e tempo de amostragem de coleta de nicotina no ambiente interno. Braga, Portugal, 2005.

\begin{tabular}{|c|c|c|c|c|}
\hline Instituição & $\begin{array}{l}\text { Locais } \\
\mathrm{N}\end{array}$ & $\begin{array}{c}\text { Amostras } \\
\mathrm{N}\end{array}$ & $\begin{array}{l}\text { Local de amos- } \\
\text { tragem }\end{array}$ & $\begin{array}{l}\text { Tempo de amos- } \\
\text { tragem }\end{array}$ \\
\hline Administração central & 1 & 4 & $\begin{array}{c}\text { Sala espera } \\
\text { Secretaria } \\
\text { Bar } \\
\text { WC }\end{array}$ & 7 dias \\
\hline Administração local & 1 & 4 & $\begin{array}{c}\text { Corredor } \\
\text { Secretaria } \\
\text { Bar } \\
\text { WC }\end{array}$ & 7 dias \\
\hline Finanças & 1 & 4 & $\begin{array}{c}\text { Corredor } \\
\text { Secretaria } \\
\text { Bar } \\
\text { WC }\end{array}$ & 7 dias \\
\hline Universidade & 1 & 4 & $\begin{array}{c}\text { Corredor } \\
\text { Secretaria } \\
\text { Bar } \\
\text { WC }\end{array}$ & 7 dias \\
\hline $\begin{array}{l}\text { Médias empresas (de } 10 \text { a } 50 \text { traba- } \\
\text { lhadores) }\end{array}$ & 4 & 16 & $\begin{array}{c}\text { Sala de espera } \\
\text { Espaço de tra- } \\
\text { balho } \\
\text { Bar } \\
\text { WC }\end{array}$ & 7 dias \\
\hline $\begin{array}{l}\text { Pequenas empresas* (com menos de } \\
10 \text { trabalhadores) }\end{array}$ & 3 & 5 & $\begin{array}{l}\text { Sala de espera } \\
\text { Zona de trabalho }\end{array}$ & 7 dias \\
\hline Restaurantes com mais de $10 \mathrm{~m}^{2}$ & 5 & 5 & Sala de jantar & 7 dias \\
\hline Restaurantes com menos de $100 \mathrm{~m}^{2}$ & 4 & 4 & Sala de Jantar & 7 dias \\
\hline Discotecas/pubs & 4 & 4 & Amostra pessoal & 3 horas \\
\hline
\end{tabular}

* Devido à reduzida dimensão de uma das empresas, apenas se colocou um monitor. 


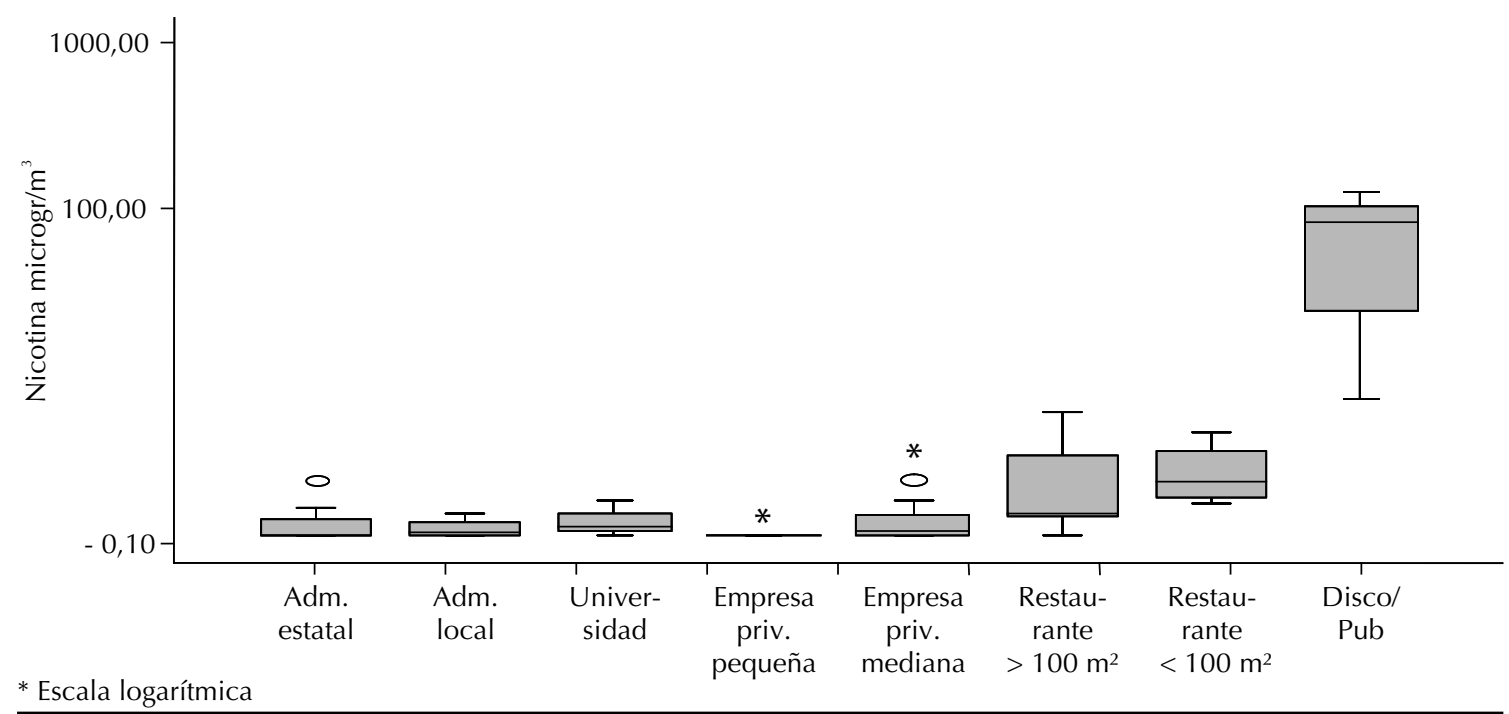

Figura. Concentração de nicotina $\left(\mu \mathrm{g} / \mathrm{m}^{3}\right)$ segundo o local estudado. Braga, Portugal, 2005.

amostrado e tipo de ventilação do local foram anotadas em fichas individuais para cada amostra. Para assegurar a qualidade da medição, após 25 amostras intercalouse um filtro não exposto ao fumo do tabaco (branco) que posteriormente era analisado para descartar a possibilidade de contaminação das amostras durante a manipulação.

Os filtros foram colocados em todos os locais com o consentimento dos responsáveis pelas instituições ou donos dos estabelecimentos, a quem foi previamente explicado os objectivos e características do estudo. Ao final foram fornecidos os resultados aos responsáveis.

O estudo obteve a aprovação do Comité Ético de Investigación Clínica del Instituto Municipal de Asistencia Sanitaria, Espanha.

\section{RESULTADOS}

A presença de nicotina foi detectada em $85 \%$ das amostras. Locais da administração pública, da administração local, das finanças e da universidade apresentaram medianas dos valores de nicotina no ar inferiores a 0,5 $\mu \mathrm{g} / \mathrm{m}^{3}$ (Figura). Nas pequenas e médias empresas, as medianas dos valores de nicotina no ar foram, de forma geral, inferiores a $0,5 \mu \mathrm{g} / \mathrm{m}^{3}$. Contudo, em algumas empresas, locais como secretarias e bares apresentaram valores apreciáveis de nicotina no $\operatorname{ar}\left(2,52 \mu \mathrm{g} / \mathrm{m}^{3}\right)$. Nos restaurantes a concentração de nicotina no ar foi mais elevada, registando-se concentrações medianas de $1,54 \mu \mathrm{g} / \mathrm{m}^{3}$ naqueles com menos de $100 \mathrm{~m}^{2}$ e de 0,37 $\mu \mathrm{g} / \mathrm{m}^{3}$ nos que possuíam uma área superior a $100 \mathrm{~m}^{2}$. Nas discotecas a concentração de nicotina foi superior à observada em todos os outros locais avaliados, com uma concentração mediana de $82,26 \mu \mathrm{g} / \mathrm{m}^{3}$, tendo-se registado o valor de $106,31 \mu \mathrm{g} / \mathrm{m}^{3}$ em uma delas.

\section{DISCUSSÃO}

Os resultados obtidos mostram que embora muitos locais públicos apresentem níveis de poluição do ar provocada pelo fumo do tabaco relativamente baixo, esta poluição é elevada em alguns locais de trabalho de médias empresas, nos restaurantes e particularmente nas discotecas.

Os níveis de exposição encontrados em discotecas são os mais extremos, chegando a alcançar concentrações de $106,31 \mu \mathrm{g} / \mathrm{m}^{3}$. Essa concentração é aproximadamente cem vezes maior que as observadas nos restaurantes, que resultam num excesso de risco extremamente significativo. As concentrações encontradas em restaurantes não chegam aos extremos em discotecas, mas são também consideravelmente altas e constituem um risco especialmente grave para os camareiros.

Em Espanha a presença de nicotina foi detectada em $92,2 \%$ das amostras. Nos locais da administração pública, a mediana dos valores de nicotina no ar foi de 0,2 $\mu \mathrm{g} / \mathrm{m}^{3}$. Nas pequenas empresas, a mediana foi inferior a $0,5 \mu \mathrm{g} / \mathrm{m}^{3}$. Nos restaurantes com menos de $100 \mathrm{~m}^{2}$ a concentração de nicotina no ar foi de $5,00 \mu \mathrm{g} / \mathrm{m}^{3}$ e nos maiores, de $3,2 \mu \mathrm{g} / \mathrm{m}^{3}$. Nas discotecas a concentração de nicotina foi superior à observada em todos os outros locais avaliados, com concentração mediana de 32,81 $\mu \mathrm{g} / \mathrm{m}^{3}$, tendo-se registado o valor de $230,32 \mu \mathrm{g} / \mathrm{m}^{3}$.

Comparando os dados obtidos em Portugal com os obtidos em Espanha, constata-se que de forma geral são semelhantes, com excepção dos restaurantes, em que os valores de nicotina obtidos em Espanha são superiores aos registrados em Portugal. Comparando os dados do presente estudo àqueles de López et al (2005), ${ }^{4,7}$ os valores de nicotina no ar são menores em Portugal do que em Barcelona (Espanha), exceto para discotecas. 
O facto da prevalência de fumadores ser maior em Espanha que em Portugal poderá, eventualmente, explicar as diferenças observadas. Estudo efectuado pelo Comité Nacional de Prevenção do Tabagismo Espanhol mostra que em amostra aleatória constituída por 2.513 pessoas de mais 18 anos, realizada em 2006, 20\% das mulheres e $27 \%$ de homens fumavam.* Em Portugal, os dados mais recentes e fiáveis são os do Inquérito Nacional de Saúde, recolhidos no ano de 1999: fumavam cerca de $29,3 \%$ dos homens e $7,9 \%$ das mulheres.**

A existência de regulamentos internos a proibir o fumo do cigarro em determinados locais (sobretudo em secretarias da administração pública) e o facto dos espaços da administração pública e local amostrados serem muitos amplos podem igualmente explicar as baixas concentrações de nicotina observadas em Portugal.

Os resultados obtidos no presente estudo permitem caracterizar com dados objectivos os níveis de fumo ambiental do tabaco a que as pessoas estão sujeitas em lugares públicos.

É imperioso controlar esta exposição, considerando que não há nenhum nível de exposição ao fumo ambiental do tabaco que se possa considerar sem risco e quanto maior for a concentração de fumo ambiental de tabaco, maior o risco para a saúde. Além disso, estar exposto durante toda a vida laboral a uma concentração de 0,7 $\mu \mathrm{g} / \mathrm{m}^{3}$ de nicotina se traduz por um excesso de risco de cancro de pulmão de 1 em 10.000. ${ }^{6}$ Isso significa que, de cada 10.000 pessoas expostas a esta concentração de nicotina durante uma vida laboral de aproximadamente 40 anos, uma desenvolveria um cancro de pulmão atribuível a esta exposição. Da mesma forma, se estiver exposto durante toda a vida laboral a uma concentração de $2,3 \mathrm{ug} / \mathrm{m}^{3}$ de nicotina provocaria um excesso de risco de cancro de pulmão de 3 em 10.000
(Repace, 1993). ${ }^{6}$

Neste contexto compreender-se-á que exposição ao FAT deva ser encarada como um grave problema de saúde pública, além das questões individuais de "estilo de vida" e da simples questão da liberdade de uso indiscriminado do tabaco em qualquer local. A única forma de proteger os não fumadores, é criando ambientes livres de tabaco, por meio de legislação.

Os dados apresentados no presente estudo confirmam a necessidade de reforçar a implementação e sobretudo o cumprimento de políticas sem fumo nos locais de trabalho e em locais públicos. Isso beneficiará, sobretudo, a saúde dos trabalhadores, com a melhoria da qualidade do ambiente de trabalho e como medida reforçadora de um ambiente que facilite aos fumadores o abandono do fumo do tabaco.

Os resultados encontrados serão importantes no processo de avaliação do impacto da lei de controlo do tabaco que vier a ser implementada em Portugal. A medição de marcadores biológicos e do ar, comparando os valores obtidos antes e depois da aplicação da lei, permitirão quantificar de maneira objectiva a diminuição dos níveis de FAT.

\section{AGRADECIMENTOS}

À Francesc Centrich, Eulàlia Serrahima e Laura Pineda, da Agencia de Salud Pública de Barcelona, pela análise das amostras; À Dr ${ }^{\text {a }}$ Rita Pinheiro e aos enfermeiros: Eurico Martins, Manuela Machado, Susana Oliveira e Paula Barbosa, alunos do mestrado de Educação para a Saúde, da Universidade do Minho, pelo apoio na realização do trabalho de campo em Portugal.

\footnotetext{
* Comité Nacional para la Prevención del Tabaquismo. Encuesta sobre conocimientos, actitudes, creencias y conductas en relación al consumo de tabaco. Madrid: 2006.

** Dias C, Martins P, Graça M. Consumo de tabaco na população de Portugal Continental: comparação dos dados dos Inquéritos Nacionais de Saúde de 1987, 1996 e 1999. Observações. Instituto Nacional de Saúde Dr. Ricardo Jorge. 2001; n¹3:3-4.
} 


\section{REFERÊNCIAS}

1. Carrión Valero F, Pellicer Ciscar C. El tabaquismo passivo en la infancia: nuevas evidencias. Prevención Tabaquismo. 2002;4(1):20-5.

2. Hammond SK. Evaluating exposure to environmental tobacco smoke. In: Winegar ED, Lawrence $\mathrm{H}$, editors. Keith Sampling and analysis of airbone pollutants. New York: Lewish; 1993. p. 319-37.

3. Hirayama T. Life-style and mortality: a large-scale cesus based cohort study in Japan. New York: Karger, 1990. Smoking and mortality; p. 54. (Contributions to epidemiology and biostatistics, 6).

4. López MJ, Nebot M, Sallés J, Serrahima E, Centrich F, Juárez $\mathrm{O}$ et al. Medición de la exposición al humo ambiental de tabaco en centros de enseñanza, centros sanitarios, medios de transporte y lugares de ócio. Gac Sanit. 2004;18(6):451-7.

5. Nebot M, López MJ, Gorini G, Neuberger M, Axelsson $\mathrm{S}$, Pilali $\mathrm{M}$ et al. Environmental tobacco smoke exposure in public places of European cities. Tob Control. 2005;14(1):60-3.
6. Repace JL, Lowrey AH. An enforceable indoor air quality standard for environmental tobacco smoke in the workplace. Risk Anal. 1993;13(4):463-75.

7. Sánchez-Martínez F, López MJ, Nebot M., Ariza C. y Grupo de Evaluación de la Ley 28/2005. Exposición al humo ambiental de tabaco en centros de trabajo previal a la entrada en vigor de la ley 28/2005 de medidas sanitarias frente al tabaquismo. Med Clin (Barc). 2007;129(3):100-3.

8. World Health Organization. International consultation on environmental tobacco smoke and child health. Geneva; 1999. [Acesso em 26 abril 2006]. Disponível em: http://www.who.int/tobacco/health_impact/youth/ ets/en/print.html

9. World Health Organization. Regional Office for Europe. Air quality guidelines for Europe. 2.ed. Copenhagen: 1987. WHO Regional Publications - European Series, 91. [Acesso em 26 abril 2006]. Disponível em: http://www.euro. who.int/document/ e71922.pdf

Estudo financiado pelo Ministerio de Sanidad y Consumo (Español) y a la Sociedad Española de Epidemiología. Projecto financiado pelo Fundo de Investigação Sanitária (Processo no PI052293). 